are common gastrointestinal medications used to manage symptoms of acid-related diseases. Studies have shown that these medications are associated with increased risk of pneumonia, vitamin deficiency, osteoporosis and fractures. Few studies have described the potential risk of inflammatory bowel disease (IBD) exacerbation among patients on gastric acid suppressants but little is known on its association. This study aims to investigate the effect of the use of gastric acid suppressants (PPI and H2RA) in the risk of IBD (Crohn's disease and ulcerative colitis) exacerbation.

Methods A comprehensive, computerized literature search from the electronic database of MEDLINE, Google Scholar, Cochrane Library, and OVID was performed with the following search terms: gastric acid suppressants, proton pump inhibitors, histamine 2 receptor antagonists, inflammatory bowel disease, Crohn's disease, ulcerative colitis, outcomes, and disease activity exacerbation. Two cohort studies were selected and validated using the Newcastle-Ottawa criteria. Trial results were combined under a random effects model using pooled relative risks (RRs). The Cochrane Review Manager Software version 5.3 was used for all analyses.

Results Two cohort studies comprising of 36,293 patients were analyzed by pooling adjusted RRs using random effects model. Disease activity exacerbation was associated with the use of gastric acid suppressants with pooled adjusted RR 1.14 [95\% CI, 1.08-1.20, I2=0\%] with no heterogeneity. The pooled adjusted RR of IBD activity exacerbation with PPI use was 1.12 [95\% CI, $1.05-1.19$, I2=0\%] for any IBD, while the pooled adjusted RR of disease activity exacerbation with HR2A use was 1.21 [1.04-1.40, I2=42\%] for IBD, with moderate heterogeneity. The effect of acid suppression was more marked in patients with Crohn's disease, RR 1.44 [0.89-2.33, I $2=77 \%$, but this was statistically insignificant with marked heterogeneity; than in ulcerative colitis RR 1.12 [1.05-1.20, $\mathrm{I} 2=0 \%$ ].

Conclusions Use of gastric acid suppressants such as PPIs and H2Ras may be associated with increased risk of disease activity exacerbation in patients with IBD. This meta-analysis confirms the need for further prospective studies in examining this relationship.

\section{IDDF2020-ABS-0095 ANALYSIS OF PREDICTIVE FACTORS FOR RO RESECTION, IMMEDIATE BLEEDING AND RECURRENCE OF COLORECTAL ADENOMAS AFTER ENDOSCOPIC MUCOSAL RESECTION}

Enrik John Aguila*, Jonard Co, Juliet Cervantes, Patricia Anne Cabral-Prodigalidad, Arsenio Caburnay, Marie Antoinette Lontok. St. Luke's Medical Center Global City, Philippines

10.1136/gutjnl-2020-IDDF.75
Background Larger colonic polyps require advanced resection techniques such as endoscopic mucosal resection (EMR) for safe and effective removal. There has been a steady accumulation of scientific evidence with regards to the technical aspects and long-term outcomes of colonic EMR compared with surgery. This study aims to determine the predictive factors of different clinical outcomes post-EMR and the diagnostic yield of JNET classification.

Methods A retrospective cohort study was done on all patients who underwent colorectal EMR at the St. Luke's Medical Center Global City within a 4-year period from 2015 to 2018. The diagnostic yield of JNET classification and clinical outcomes namely R0 resection, complications and recurrence of lesions were studied.

Results A total of 282 patients were studied. The R0 resection rate was $96.3 \%$ for lesions resected en bloc. $15.2 \%$ had a complication, most commonly intraprocedural bleeding which were successfully managed endoscopically. $10.7 \%$ had recurrence post-EMR on their surveillance colonoscopy. The JNET classification exhibited good sensitivity for Type 1 $(71.8 \%)$ and Type 2A (91.9\%) and good specificity for Type 1 (96.9\%) and Type 2B (95.5\%). Accuracy was high at $91.02 \%$ for Type $1,80.24 \%$ for Type $2 \mathrm{~A}$ and $89.22 \%$ for Type 2B.

Conclusions EMR is an important advancement in the field of therapeutic endoscopy with good clinical outcomes sparing patients from surgery. A larger lesion size of $>20 \mathrm{~mm}$ is associated with both positive resection margin and post-EMR complications. Main predictors of recurrence include a nongranular morphology of a resected polyp and piecemeal resection. The JNET classification has a high diagnostic accuracy rate; hence is a good endoscopic tool for characterization of lesions.

\section{IDDF2020-ABS-0096 CLINICAL STUDY ON SIMPLIFIED ENDOSCOPIC SUBMUCOSAL DISSECTION FOR COLORECTAL LATERALLY SPREADING TUMORS}

Xiao-qiao Yang*. Guangdong Second Provincial General Hospital, China

\subsection{6/gutjn|-2020-IDDF.76}

Background To evaluate the efficacy and safety of Simplified Endoscopic Submucosal Dissection (SESD) for colorectal laterally spreading tumors (LSTs).

Methods Patients of colorectal laterally spreading tumors (LSTs) with a diameter of over $2 \mathrm{~cm}$ from January 2017 to January 2019 were enrolled in our study. Patients were assigned to 2 groups according to endoscopy procedures, the SESD group and ESD group.SESD includes three steps, 1) pre-cut circularly around the tumors, 2) dissected the

Abstract IDDF2020-ABS-0095 Table 1 Risk factors associated with clinical outcomes post-EMR

\begin{tabular}{ll}
\hline Positive Resection Margin & Complications (Bleeding or Perforation) \\
\hline - Gross lesion size $>20 \mathrm{~mm}$ & - Use of hybrid EMR technique \\
- Presence of submucosal fibrosis & - Gross lesion size $>20 \mathrm{~mm}$ \\
- Histopathologic size $>20 \mathrm{~mm}$ & - Non-granular morphology of a laterally spreading tumor \\
- Moderately differentiated adenocarcinoma on histopathology & - Histopathologic size of $>20 \mathrm{~mm}$ \\
& - Use of saline and methylene blue as lifting agents \\
\hline
\end{tabular}

